\title{
Systemic Consolidation Treatment after (Chemo) Radiotherapy in Rectal Cancer Treatment
}

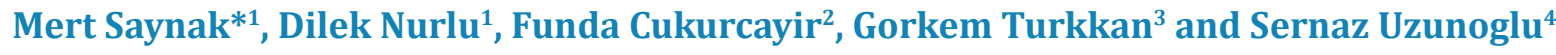 \\ ${ }^{1}$ Department of Radiation Oncology, Trakya University Hospital, Turkey \\ ${ }^{2}$ Department of Radiation Oncology, Antakya State Hospital, Turkey
}

${ }^{3}$ Department of Radiation Oncology, Edirne State Hospital, Turkey

${ }^{4}$ Department of Medical Oncology, Trakya University Hospital, Turkey

Received: March 26, 2018; Published: April 12, 2018

*Corresponding author: Mert Saynak, Department of Radiation Oncology, Trakya University Hospital, 22030, Edirne, Turkey, Tel: 00902842361074;

Email: mertsaynak@trakya.edu.tr

\author{
Abbreviations: ChT : Chemotherapy; RT: Radiotherapy; pCR: Complete Response; MRI: Magnetic Resonance Images; PET: Positron Emission \\ Tomography; CT: Computed Tomography
}

\section{Introduction}

Most people with locally advanced rectal cancer are treated with chemotherapy (ChT), radiotherapy (RT), and surgery. With improved surgical techniques and the addition of neoadjuvant RT区 ChT, 5-year local recurrence rates have decreased from $>25 \%$ to $<10 \%$ [1,2]. Regarding to local/regional disease site, quality of life is a new and increasingly important goal for rectal cancer treatment. However, these advances have not appreciably decreased the risk of distant metastatic recurrence (approximately 30\%), which remains the leading cause of rectal cancer-related death. That's why, systemic treatment to control distant microscopic disease especially has become increasingly importance in recent years.

Preoperative radiotherapy has an accepted role in reducing the risk of local recurrence in locally advanced rectal cancer, particularly when the circumferential resection margin is breached or threatened. There are several reports about patients who have neoadjuvant ChRT and surgery is not tolerating well the adjuvant chemotherapy. Whereas, the consolidation chemotherapy strategy has the potential to increase local disease control and distant control with relatively good treatment compliance. We would like to glance at pros and cons about total neoadjuvant treatment and nonsurgical treatment in this paper.

\section{What is the Importance of Complete Response?}

Neoadjuvant chemo radiotherapy (ChRT) has the potential benefit to improve of local disease control. Besides it may provide significant tumor downsizing and down staging and results in pathological complete response (pCR) in $\nabla 15-30 \%$ of the cases [3]. The data that have been accumulated so far show that, patients who achieved pCR after CRT have better long-term outcomes, less propensity to develop local and distal recurrence, and improved survival [1]. One assumption proposed to explain this issue suggests those cases with pCR have relatively early stage and small disease initially. Another explanation is that obtaining a complete response in the local disease with CRT may indicate that the tumor is sensitive to $\mathrm{ChT}$, indicating that it may be a controlled systemic disease. Total neoadjuvant therapy can be designed as chemotherapy followed by RT $(\otimes \mathrm{ChT}$ ) in order to prolong the waiting period until surgery. Thus, by this treatment method, response probability can be increased for a couple of reason:

a. $\quad$ Longer waiting period and

b. Additional chemotherapy courses through this waiting period.

According to the data obtained from several prospective, singlearm studies that had $>90 \%$ consolidation ChT completion rates $[4,5]$. Several ways have been tried to increase the likelihood of providing tumor response. One approach is to lengthen the interval between ChRT and surgery, because it has been proven that pCR is time-dependent. French study (Lyon R90-01) demonstrated that $\geq 6$ weeks after RT has better response outcomes [5,6]. Actually, optimal time to surgery is still debated. A couple of randomized studies were carried out to compare 6 vs 12 weeks and 7 vs 11 weeks after ChRT. As a result, 12 weeks waiting period looked 
better than 6 weeks for pCR, but 7 and 11 weeks had similar pCR rates with higher surgical morbidity [7]. As the time to surgery increases, the concern that RT will cause an increase in late effects, which will increase the morbidity of the surgical procedure, has not been confirmed in other studies conducted in this area [8]. The second is the integration of different systemic treatment schemes into preoperative radiotherapy. Disappointingly, the addition of oxaliplatin to standard concurrent ChRT protocol was shown to increase toxicity risk without improving tumor response in most of the phase III randomized studies [9-11].

Only the German study showed an improvement in pCR in an unplanned exploratory analysis [12]. Duke University reported encouraged results with four cycles of bevacizumab infusion concurrently with $50.4 \mathrm{~Gy} \mathrm{RT}$ and two cycles of 24-hour continuously infused 5-fluorouracil. In their phase I/II clinical study of 32 patients, they showed that $100 \%$ local control with this treatment schema [13]. Retrospective studies imply brachytherapy boost may improve the response possibility [14,15]. Besides the Lyon R96.02 Randomized Study demonstrated if contact 50 kilovolt X-ray treatment was administered in addition to external RT, the probability of pathologic complete response and in 10 years' follow-up, colostomy-free survival was increased [16]. If brachytherapy is administered alone without external radiotherapy, it is disadvantageous that the mesorectal lymph nodes receive an insufficient dose, and even that the lateral lymph nodes receive almost no dose. As systemic treatment options progress, brachytherapy may play a more central role in the treatment of rectal cancer.

\section{Consolidation Treatment After (Chemo) Radiotherapy}

Another strategy for obtaining tumor response is to administer additional ChT during the waiting period between ChRT and surgery. One study demonstrated that 3 additional cycles FOLFOX after standard ChRT with fluoroprimidin-based ChT improved tumor response. In this prospective study, 34 patients with rectal cancer underwent 5-fluorouracil-based chemotherapy every 21 days in totally six cycles. The authors concluded, although in a preliminary basis, that the addition of chemotherapy after ChRT during the resting period (also known as "consolidation" chemotherapy) resulted in considerably high rates of complete response [17]. Recently, a study reported from United States, in which the primary study endpoint was the pCR rate. Remarkably, pCR rates increased significantly with additional cycles of FOLFOX between ChRT and surgery, with $38 \%$ of patients achieving pCR when 6 cycles of ChT were given after CRT (compared with an 18\% pCR rate for patients who did not receive ChT between CRT and surgery) [18].

The Polish Colorectal Study Group conducted a phase III randomized study including 515 patients with unrespectable locally advanced rectal cancer (stage cT3 or cT4) to compare the results of short and long course RT schemas. In the control arm, patients received radiotherapy at 50.4 Gy given concurrently over approximately 5 weeks with bolus fluorouracil (5-FU) and leucovorin during the first and fifth weeks, plus oxaliplatin at 50 $\mathrm{mg} / \mathrm{m}^{2}$ once weekly. The patients in experimental arm received 5 x 5 Gy RT followed by three cycles of FOLFOX4 (5-FU, leucovorin, oxaliplatin) every 2 weeks. R0 resection, the primary endpoint, was achieved in $77 \%$ of the experimental arm and $71 \%$ of the control arm $(\mathrm{p}=0.07)$, indicating a trend in favor of the short-course approach. Pathologic complete responses were achieved by $16 \%$ and $12 \%$, respectively $(p=0.17)$ [19]. The results of a phase II randomized trial, in which the efficacy of ChT with bevacizumab in the waiting period after ChRT is tested, are expected to understand the rationale for the non-surgical treatment approach be released soon [20].

The Korean Society of Coloproctology (KONCLUDE Study) has started a prospective randomized study to evaluate additional 3 cycles of chemotherapy consisted with FOLFOX regimen between neoadjuvant ChRT and surgery. Their expectance that this treatment approach could increase the proportion of pathologic complete response ( $\mathrm{pCR}$ ), subsequently could show better oncologic outcomes [21]. Similarly, impressive treatment compliance rates have been observed in studies using the second total neoadjuvant treatment paradigm-induction ChT followed by ChRT and then surgery. However, there is not so much high level evidence about superiority of this approach [22-25]. On the other hand, some patients initially have obstruction and hemorrhagic findings due to big tumors. That's why, starting with RT instead of systemic therapy alone, may provide a kind of early palliation theoretically and performing chemotherapy before the surgery instead of postoperative period improves patient compliance to systemic therapy, and increased tumor response probability. The recommended treatment strategy is shown diagrammatically in (Figure 1).

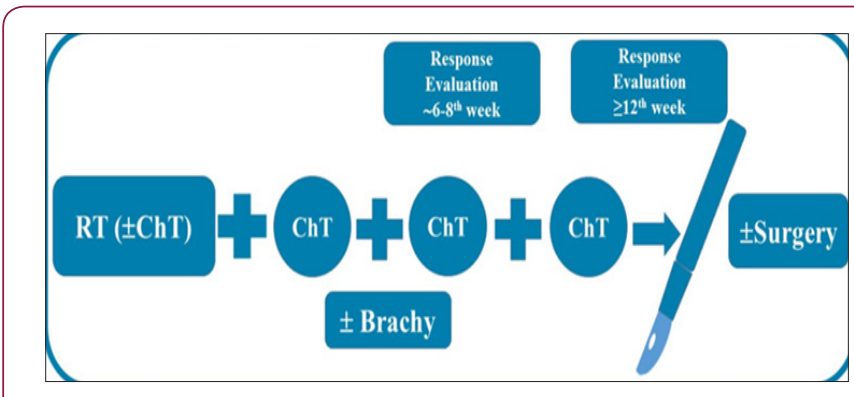

Figure 1: The recommended treatment treatment strategy is shown diagrammatically.

Note: RT: Radiotherapy, Cht: Chemotherapy, Brachy: Brachytherapy

\section{Rationale for Non-Surgical Treatment Approach}

It's clear that patients treated using non-surgical approach because of achieved complete tumor response is avoided from the risk of surgical mortality and morbidity on urinary, fecal and sexual functions. The studies have been published so far showed that, the oncologic outcome after non-surgical approach was comparable with that of patients with a pCR after surgery, but functional outcome was significantly better [26,27]. Long term results of non-surgical approach have been firstly reported by Brazilian investigators. A total of 99 patients had a sustained cCR for $\geq 1$ year 
and managed non-surgically. They reported 13 recurrences (13\%) and 5 isolated recurrences were effectively salvaged at a mean 60 months' follow-up. They reported that 5 isolated endorectal recurrences were effectively salvaged. In the end, 5-year overall survival and disease-free survival rates were $93 \%$ and $85 \%$, respectively [26]. Researchers in Holland initiated a prospective study. Twenty-one patients with cCR were managed non-surgically. As a result, only one patient developed end luminal recurrence and underwent surgical salvage [27]. Recently, a propensity matched cohort study(the OnCoRe (Outcomes after Clinical Complete Response in Patients with Rectal Cancer)) study evaluating nonsurgical treatment have been published from United Kingdom. 259 patients were included of which 228 underwent surgery. Although this sounds like a potentially attractive option, only 12\% (31 of 259) of the patients in this study experienced a complete response. As a result, there was no difference between the matched paired groups [28].

\section{Disadvantages of Non-Surgical Treatment Approach}

Unfortunately, major clinical response is not easy to define and not truly reliable in predicting pathological complete response (pCR). There is a difficulty to distinguish between tumor and fibrosis on magnetic resonance images (MRI) taken for restaging. Positron emission tomography (PET) is advantageous over computed tomography (CT) and MRI in that the latter two imaging modalities are often limited in differentiating post-treatment fibrosis from residual or recurrent tumor. Detection of residual tumor cell clusters using non-surgical methods is still not entirely possible. In the group of patients who underwent surgery after ChRT, 25-77\% of patients with clinical complete response (cCR) had pCR [29-31]. At the current time, using the latest available imaging diagnostic techniques, we still have always a risk of misdiagnosing about 15$20 \%$ with the most optimistic estimation in identifying pCR.

Question about the way in which patients who has achieved a true pCR to identify with precision is still being evaluated. Some clinicians suggest full thickness local excision for this patient group with cCR. The arguments of this approach are, it can be a treatment of residual disease at a certain level and can be helped to define pathological response as good as $99 \%$ for T stage. However, complete response of the primary tumor cannot predict response in regional lymph nodes, which are involved in 7 to $17 \%$ of patients who have cCR of the primary tumor [32]. Theoretically, anastomosis problems may constitute a potential disadvantage of this approach compared to non-surgical treatment. The 45-54 Gy dose is lower than the curative dose used by radiation oncologists in other diseases like head and neck cancers, lung cancers, endometrial and cervical cancers. There are some question marks about the progression-free time that the dose in question can provide. In the studies evaluated non-surgical approach has been used longcourse radiotherapy.

Both the Dutch and Swedish rectal cancer studies used neoadjuvant short-course radiotherapy in 5 x 5 Gy and patients typically underwentsurgical resection within 1 week of radiotherapy completion. This approach looks effective to reduce local recurrence risk but in the past tumor regression was not primary aim of short course radiotherapy. However, the Poland Group conducted a prospective randomized study and demonstrated that 5 x 5 Gy followed by 3 cycles of FOLFOX and relatively long time waiting to surgery provides similar response with long course ChRT [19]. In rectal cancer population there is a kind of nasty local recurrence shape which may be involved to sacral nerves and attached to sacral bone. Even when the mesorectal is removed by performing total mesorectal excision, presacral region is the most important local recurrence site. Since the tumor cells can easily progress through sacral nerves into the sacral bone. This is a kind of nightmare for the clinicians.

Because it's a very painful clinical manifestation for the patient and surgical salvage is highly difficult in many such cases. Even though impressive initial study results with non-surgical approach reported by Brazil and Holland, local recurrence rate may be reach about $25 \%$. However, local recurrences can be salvaged successfully if the close follow-up strategies are applied [33]. In relatively early stages, small tumors like small T3 a,b N0 disease, complete response may more possible than relatively more advanced stages like T4 and lymph node(s) clinically positive disease. However, for the T3a,b disease the recent wisdom for the management of rectal cancer treatment if TME is achievable, recommends surgery initially. Because postoperative ChRT could be selectively used in such patients [34] Therefore, the patient group that is more likely to achieve complete response with neoadjuvant therapy will not have received ChRT for this purpose.

In today's modern era, it is clear that the common standards for large heterogeneous patient groups need to be replaced by more individualized therapies. Non-surgical treatment for complete responders after ChRT for rectal cancer should be based on strict selection and follow-up with endoscopy and up-to-date imaging may be practicable. Even so, the decision to undergo to surgery is up to patients' willingness. Patients should be informed that non-surgical approach remains unproven yet31. If a patient who had clinical CR volunteers to accept some risk to postpone rectal surgery in hope of avoiding major surgery and preserving rectal function can be taken a close follow-up protocol. The effective and safe implementation of this approach requires a multidisciplinary approach and very close cooperation between these different disciplines.

\section{Conclusion}

For many years the primary focus of rectal cancer treatment has been local/regional pelvic disease control. With current surgical methods and modern advanced techniques, the risk of local recurrence has been reduced to less than $10 \%$.Thus reducing the risk of developing distant metastases and increasing the quality of life of surviving patients should be new aims of treatment. Theoretically, the patients who have the increased cCR provided by "prolonged waiting time and extra chemotherapy courses" will have the option of follow-up without surgery. This approach may allow for non-surgical treatment and so may save the patients from the comorbidities associated with surgical intervention. Besides, even if resection is to be done, completion of ChTprior to surgery 
can also ensure that patients complete the ChT regimen. Of course; these theoretical assumptions should be supported by phase III randomized study results.

\section{References}

1. MacFarlane JK, Ryall RD, Heald RJ (1993) Mesorectal excision for rectal cancer. Lancet 341(8843): 457-460.

2. Sauer R, Becker H, Hohenberger W, Claus Rödel, Christian Wittekind, et al. (2004) Preoperative versus postoperative chemo radiotherapy for rectal cancer. N Engl J Med 351:1731-1740.

3. Maas M, Nelemans PJ, Valentini V, Das P, Rödel C, et al. (2010) Longterm outcome in patients with a pathological complete response after chemoradiation for rectal cancer: a pooled analysis of individual patient data. Lancet Oncol 11(9): 835-844.

4. Gao YH, Zhang X, An X, Cai MY, Zeng ZF, et al. (2014) Oxaliplatin and capecitabine concomitant with neoadjuvant radiotherapy and extended to the resting period in high risk locally advanced rectal cancer. Strahlenther Onkol 190(2): 158-164.

5. Zampino MG, Magni E, Leonardi MC, Petazzi E, Santoro L, et al. (2009) Capecitabine initially concomitant to radiotherapy then perioperatively administered in locally advanced rectal cancer. Int J Radiat Oncol Biol Phys 75(2): 421-427.

6. Francois Y, Nemoz CJ, Baulieux J, Vignal J, Grandjean JP, et al. (1999) Influence of the interval between preoperative radiation therapy and surgery on downstaging and on the rate of sphincter-sparing surgery for rectal cancer: the Lyon R90-01 randomized trial. J Clin Oncol 17(8): 2396.

7. Lefevre JH, Mineur L, Kotti S, Rullier E, Rouanet P, et al. (2016) Effect of Interval (7 or 11 weeks) Between Neoadjuvant Radiochemotherapy and Surgery on Complete Pathologic Response in Rectal Cancer: A Multicenter, Randomized, Controlled Trial (GRECCAR-6). J Clin Oncol 34(31): 3773-3780.

8. Foster JD, Jones EL, Falk S, Cooper EJ, Francis NK (2013) Timing of surgery after long-course neoadjuvant chemoradiotherapy for rectal cancer: a systematic review of the literature. Dis Colon Rectum 56(7): 921-930.

9. Gerard JP, Azria D, Gourgou Bourgade S, Martel Laffay I, Hennequin C, et al. (2010) Comparison of two neoadjuvant chemoradiotherapy regimens for locally advanced rectal cancer: results of the phase III trial ACCORD 12/0405-Prodige 2. J Clin Oncol 28(10): 1638-1644.

10. Aschele C, Cionini L, Lonardi S, Pinto C, Cordio S, et al. (2011) Primary tumor response to preoperative chemoradiation with or without oxaliplatin in locally advanced rectal cancer: pathologic results of the STAR-01 randomized phase III trial. J Clin Oncol 29(20): 2773-2780.

11. O'Connell MJ, Colangelo LH, Beart RW, Petrelli NJ, Allegra CJ, et al. (2014) Capecitabine and oxaliplatin in the preoperative multimodality treatment of rectal cancer: surgical end points from National Surgical Adjuvant Breast and Bowel Project trial R-04. J Clin Oncol 32(18): 19271934.

12. Rodel C, Liersch T, Becker H, Fietkau R, Hohenberger W, et al. (2012) Preoperative chemoradiotherapy and postoperative chemotherapy with fluorouracil and oxaliplatin versus fluorouracil alone in locally advanced rectal cancer: initial results of the German CAO/ARO/AIO-04 randomised phase 3 trial. Lancet Oncol 13(7): 679-687.

13. Willett CG, Duda DG, di Tomaso E, Boucher Y, Ancukiewicz M, et al. (2009) Efficacy, safety, and biomarkers of neoadjuvant bevacizumab, radiation therapy, and fluorouracil in rectal cancer: a multidisciplinary phase II study. J Clin Oncol 27(18): 3020-3026.

14. Sun Myint A, Mukhopadhyay T, Ramani VS, Perkins K, Snee AJ, et al. (2010) Can increasing the dose of radiation by HDR brachytherapy boost following pre-operative chemoradiotherapy for advanced rectal cancer improve surgical outcomes? Colorectal Dis 12(2): 30-36.
15. Rijkmans EC, Cats A, Nout RA, van den Bongard DHJG, Ketelaars M, et al. (2017) Endorectal Brachytherapy Boost After External Beam Radiation Therapy in Elderly or Medically Inoperable Patients With Rectal Cancer: Primary Outcomes of the Phase 1 HERBERT Study. Int I Radiat Oncol Biol Phys 98(4): 908-917.

16. Gerard JP, Chapet O, Nemoz C, Hartweig J, Romestaing P, et al. (2014) Improved sphincter preservation in low rectal cancer with high-dose preoperative radiotherapy: the lyon R96-02 randomized trial. J Clin Oncol 22(12): 2404-2409.

17. Habr Gama A, Perez RO, Sabbaga J, Nadalin W, São Julião GP, et al. (2009) Increasing the rates of complete response to neoadjuvant chemoradiotherapy for distal rectal cancer: results of a prospective study using additional chemotherapy during the resting period. Dis Colon Rectum 52(12): 1927-1934.

18. Garcia Aguilar J, Chow OS, Smith DD, Marcet JE, Cataldo PA, et al. (2015) Effect of adding mFOLFOX6 after neoadjuvant chemoradiation in locally advanced rectal cancer: a multicentre, phase 2 trial. Lancet Oncol 16(8): 957-966.

19. Bujko K, Wyrwicz L, Rutkowski A, Malinowska M, Pietrzak L, et al. (2016) Long-course oxaliplatin-based preoperative chemoradiation versus $5 \mathrm{x}$ 5 Gy and consolidation chemotherapy for cT4 or fixed cT3 rectal cancer: results of a randomized phase III study. Ann Oncol 27(5): 834-842.

20. Glynne Jones R, Hava N, Goh V, Bosompem S, Bridgewater J, et al. (2015) Bevacizumab and Combination Chemotherapy in rectal cancer Until Surgery (BACCHUS): a phase II, multicentre, open-label, randomised study of neoadjuvant chemotherapy alone in patients with high-risk cancer of the rectum. BMC Cancer 15: 764.

21. Chang Woo Kim, Ik Yong Kim, Suk Hwan Lee (2010) Consolidation chemotherapy for locally advanced mid- or low rectal cancer after neoadjuvant concurrent chemoradiotherapy: A multicenter, randomized controlled trial (KONCLUDE). Journal of Clinical Oncology.

22. Gao YH, An X, Sun WJ, Cai J, Cai MY, et al. (2014) Evaluation of capecitabine and oxaliplatin administered prior to and then concomitant to radiotherapy in high risk locally advanced rectal cancer. J Surg Oncol 109(5): 478-482.

23. Marechal R, Vos B, Polus M, Delaunoit T, Peeters M, et al. (2012) Short course chemotherapy followed by concomitant chemoradiotherapy and surgery in locally advanced rectal cancer: a randomized multicentric phase II study. Ann Oncol 23(6): 1525-1530.

24. Dipetrillo T, Pricolo V, Lagares Garcia J, Vrees M, Klipfel A, et al. (2012) Neoadjuvant bevacizumab, oxaliplatin, 5-fluorouracil, and radiation for rectal cancer. Int J Radiat Oncol Biol Phys 82(1): 124-129.

25. Schou JV, Larsen FO, Rasch L, Linnemann D, Langhoff J, et al. (2012) Induction chemotherapy with capecitabine and oxaliplatin followed by chemoradiotherapy before total mesorectal excision in patients with locally advanced rectal cancer. Ann Oncol 23(10): 2627-2633.

26. Habr Gama A, Perez RO, Nadalin W, Sabbaga J, Ribeiro U, et al. (2004) Operative versus nonoperative treatment for stage 0 distal rectal cancer following chemoradiation therapy: long-term results. Ann Surg 240(4): 711-717.

27. Maas M, Beets Tan RG, Lambregts DM, Lammering G, Nelemans PJ, et al. (2011) Wait-and-see policy for clinical complete responders after chemoradiation for rectal cancer. J Clin Oncol 29(35): 4633-4640.

28. Renehan AG, Malcomson L, Emsley R, Simon Gollins, Andrew Maw, et al. (2016) Watch-and-wait approach versus surgical resection after chemoradiotherapy for patients with rectal cancer (the OnCoRe project): a propensity-score matched cohort analysis. Lancet Oncol 17(2): 174183.

29. Lambregts DM, Vandecaveye V, Barbaro B, Bakers FC, Lambrecht M, et al. (2011) Diffusion-weighted MRI for selection of complete responders after chemoradiation for locally advanced rectal cancer: a multicenter study. Ann Surg Oncol 18(8): 2224-2231. 
30. Ferrari L, Fichera A (2015) Neoadjuvant chemoradiation therapy and pathological complete response in rectal cancer. Gastroenterol Rep (Oxf) 3(4): 277-288.

31. Barbaro B, Vitale R, Valentini V, Illuminati S, Vecchio FM, et al. (2012) Diffusion-weighted magnetic resonance imaging in monitoring rectal cancer response to neoadjuvant chemoradiotherapy. Int J Radiat Oncol Biol Phys 83(2): 594-599.

32. Hughes R, Glynne Jones R, Grainger J, Richman P, Makris A, et al. (2006) Can pathological complete response in the primary tumour following pre-operative pelvic chemoradiotherapy for T3-T4 rectal cancer predict for sterilisation of pelvic lymph nodes, a low risk of local recurrence and the appropriateness of local excision? Int J Colorectal Dis 21(1): 11-17.

33. Van der Valk MJM, Hilling DE, van de Velde CJH (2017) What is there to learn for watch-and-wait in rectal cancer? Lancet Gastroenterol Hepatol 2(7): 467-468.

34. Glynne Jones R, Wyrwicz L, Tiret E, Brown G, Rödel C, et al. (2017) Rectal cancer: ESMO Clinical Practice Guidelines for diagnosis, treatment and follow-up. Ann Oncol 28(4): iv22-iv40.

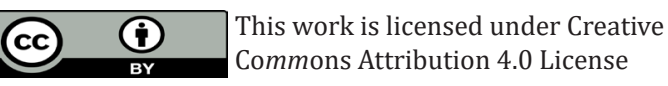

Submission Link: https://biomedres.us/submit-manuscript.php

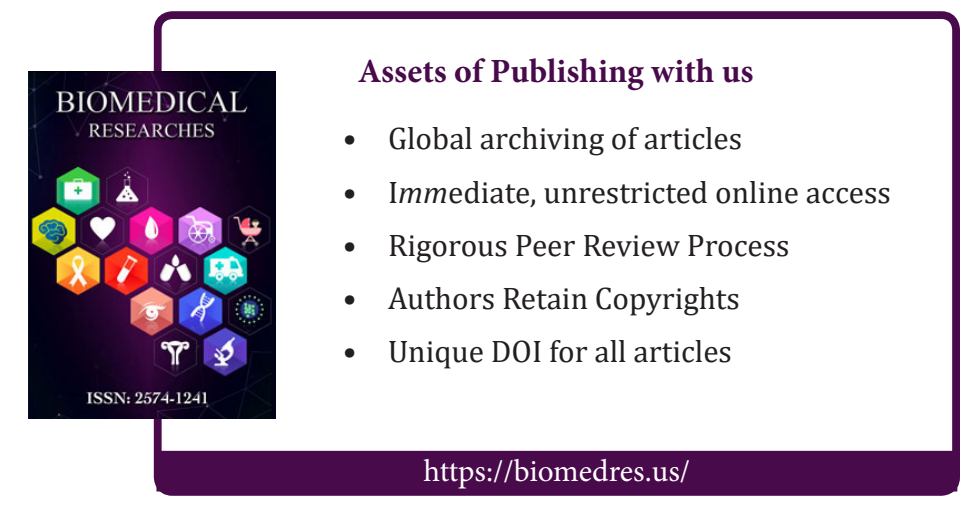

